\title{
Task-Oriented Petri Net Models for Discrete Event Simulation
}

\author{
Ewa Ochmanska \\ Warsaw Technical University, Department of Transport \\ 00-662 Warsaw, Poland \\ ochait.pw.edu.pl
}

\begin{abstract}
The paper concerns a class of task-oriented simulation models for discrete event systems. Systems are considered, in which arriving tasks are executed in alternative ways, according to the actual state of particular system resources. The models are based on Petri nets, with extensions including informative aspects of modeled processes as well as timing and decisionmaking rules. Model structure and behavior conform to realities of railway control. Similar models can be helpful in investigating different types of systems executing tasks by means of distributed sets of resources with dynamically changing availability. In particular they are suitable for modeling reliability behavior of control systems, built of re-configurable modules.
\end{abstract}

\section{Introduction}

A discrete event simulation model, presented in the paper, propagates tasks arriving to modeled system through the chain of task executors. Tasks are executed in one of possible ways or discarded, according to the current state of system resources. Such a model can be applied in investigating system reliability or performance.

General assumptions for system structure and functions, taken from realities of railway station control system, lead to the task-oriented modeling principle with dynamically changing states of resources needed for alternative task executions, formulated in section 2 . The model represents processes of execution of tasks arriving to the system by occupying one of several predefined configurations of resources with limited and changing availability, corresponding to failures of technical devices [3]. Timed nets with data structures, predicates and actions [2,4], defined upon place/transition Petri nets [1], were applied earlier and proved as effective tool for event-oriented modeling and simulation of technological processes at railway stations $[4,6]$ and processes of transport \& distribution in logistic systems [5]. The new feature of models presented in this paper consists in task- versus event-oriented view of the system rather than in net model description. The formalism of Petri net extension adopted for this kind of models is shortly resumed in section 3 .

In section 4, the composition and dynamics of net elements of simulation model are explained, as well as general structure and behavior of the model. Section 5 contains final remarks concerning some problems, which need resolving, and possible applications of the presented models. 


\section{Task-Oriented View of a System}

\subsection{System Resources}

We assume that a control part $\mathbf{C}$ of modeled system disposes resources of a set $\mathbf{R}$ as a means to execute a stream of tasks, i.e. a sequence of tasks incoming to the system at successive points of time. All resources are divided into $n$ disjoint subsets:

$$
\mathbf{R}=\left\{\mathbf{r}_{f}, f=1, \ldots, p\right\}=\mathbf{R}_{1} \cup \ldots \cup \mathbf{R}_{n},
$$

which are physically distributed and joint to the control part by communication links. The distributed subsets of resources form a family:

$$
\mathbf{R}=\left\{\mathbf{R}_{i}, i=1, \ldots, n\right\} .
$$

At a given point of time, each individual resource $\mathbf{r} \in \mathbf{R}$ may be in one of the following states: $1^{\circ}$ available: being ready to use; $2^{\circ}$ occupied: participating in execution of a task; $3^{\circ}$ unable: malfunctioning; $4^{\circ}$ inaccessible because of malfunction of its communication link (this case simultaneously concerns all resources from a set $\mathbf{R}_{i}$ ).

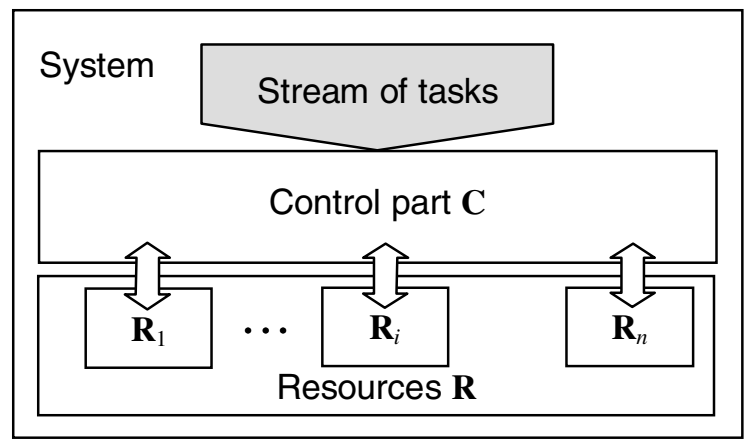

Fig. 1. Task-oriented view of the modeled system

The control part $\mathbf{C}$ of the system checks actual states of particular resources and distributes, through communication links, execution of incoming tasks to available resources proper to specific kinds of tasks, according to definitions formulated in the next subsection.

\subsection{Executive Configurations of Resources}

For execution of a task, some subset of system resources is needed for some period of time. Such subset of resources will be called executive configuration of a task. A finite family of different executive configurations, applied for execution of all system tasks, is a finished family of $m$ resource subsets:

$$
\mathbf{E}=\left\{\mathbf{E}_{k} \subseteq \mathbf{R} \mid k=1, \ldots, m\right\} .
$$

In general, an executive configuration of a task may be distributed, i.e. may contain resources of different subsets of family $\mathbf{R}$. 
A task can be alternatively executed in more than one configuration of resources, e.g. a train coming to the station can be placed on different tracks with the use of different control devices. Moreover, the modeled system executes tasks of different kinds, e.g. a station sends trains of various lengths to different directions. The kinds of tasks are distinguished by sets of possible executive configurations and their kindspecific time characteristics (duration of execution and occupation of resources).

All kinds of tasks executed by the system are described by a set $\mathbf{K}$ of pairs:

$$
\mathbf{K}=\left\{\mathbf{K}_{j}=\left|\mathbf{E}_{j}, \boldsymbol{\mathcal { T }}_{j}\right\rangle \mid j=1, \ldots, q\right\},
$$

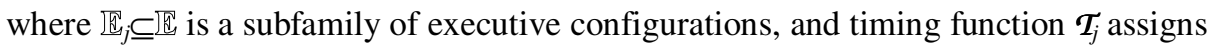
a time period of being occupied by executing a task of $j$-th kind to each particular resource of each particular configuration in $\mathbb{\Theta}_{j}$.

Accordingly to (4), different executive configurations may be alternatively applied to a task of given kind. On the other hand, the same executive configuration may be applicable to multiple kinds of tasks, i.e. families of configurations proper to different kinds of tasks may have common parts. However, timing functions are defined independently and the same configuration of resources may be applied for different kinds of tasks with different time characteristics.

Let us order executive configurations proper to a task of $j$-th kind from most to less convenient, and appropriately enumerate elements of subfamily $\mathbb{\Omega}_{j}$ :

$$
\mathbf{E}_{j}=\left\{\mathbf{E}^{j d} \in \mathbf{E}\right\}_{d=1, \ldots,|\mathbf{E} j|},
$$

where $\left|\mathbb{E}_{j}\right| \geq 1$ is cardinality of the set $\mathbb{\mathbb { A }}_{j}$.

An executive configuration proper to multiple kinds of tasks may have different order for each of those kinds.

\subsection{An Algorithm for System Dynamics}

An executive configuration, chosen by the control part of a system for executing a task, is called its active configuration. During execution of a task of $j$-th kind, each resource in its active configuration is occupied by a period of time determined by the timing function $\mathcal{T}_{j}$. A resource may be used for execution of one task at a time, i.e. occupied by at most one active configuration.

The tasks arrive to the input of the modeled system in some distinguished points of time. The control part of the system attempts to execute them in configurations of lowest possible order, while the states of resources change dynamically and not all of them are available. Some of resources are occupied by active configurations. Some of them have failed or became inaccessible because of damaged communication links. Assigning an active configuration for a task of $j$-th kind is done by searching through all executive configurations of the system, starting from the configuration $\mathbf{E}^{j 1}$ first in order for this kind of tasks.

Let us consider a task incoming to a system, which remains at a given moment in some current state of resources. We will distinguish five following cases with regard to possibility of executing a task of $j$-th kind in executive configuration $\mathbf{E}_{k} \in \mathbb{E}$ as the configuration of $d$-th order $\mathbf{E}^{j d} \in \mathbb{\mathbb { E }}_{j}, d \in 1, \ldots,\left|\mathbb{\mathbb { E }}_{j}\right|$, proper to that kind of tasks: 
(a) $\quad \mathbf{E}_{k} \neq \mathbf{E}^{j d}$. Another configuration $\mathbf{E}_{l} \in \mathbb{\mathbb { N }}, l \neq k$ and $\mathbf{E}_{l}=\mathbf{E}^{j d}$, should be considered.

(b) $\mathbf{E}_{k}=\mathbf{E}^{j d}$ and all its resources are available.

The task is executed in active configuration $\mathbf{E}_{k}$. The resources belonging to that configuration are occupied for a time given by the timing function $\mathcal{T}_{j}$.

(c) $\mathbf{E}_{k}=\mathbf{E}^{j d}$, but it is not possible to activate this configuration, because some of its resources are occupied, i.e. participating in an active configuration.

The task is waiting to be executed in the configuration $\mathbf{E}_{k}$ after releasing occupied resources.

(d) $\mathbf{E}_{k}=\mathbf{E}^{j d}$, but it is not possible to activate this configuration because of malfunctions of some elements of the system: one or more resources belonging to $\mathbf{E}_{k}$ are in states of inability or inaccessibility. Besides, $d<\left|\mathbb{\mathbb { E } _ { j }}\right|$, i.e. there is an

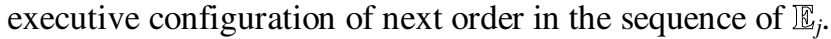

Then the control part of the system attempts to activate some configuration $\mathbf{E}_{l} \in \mathbb{\mathbb { A }}, l \neq k$, as the next executive configuration $\mathbf{E}^{j(d+1)}$ proper to the kind of task.

(e) $\mathbf{E}_{k}=\mathbf{E}^{j d}$, but it is not possible to activate this configuration because one or more resources belonging to $\mathbf{E}_{k}$ are in states of inability or inaccessibility, and $d=\mid \mathbb{E}_{j}$ l, i.e. $\mathbf{E}^{j \mathrm{~d}}$ is last in the sequence of $\mathbb{\circledR}_{j}$.

The task can not be executed in a regular way. For such cases we may define several treatments depending on specifics of modeled problem, e.g. refusing execution, waiting for recovering of needed resources or suspending the system normal activity for a period of time consumed by special procedures.

\section{Net Tools for Model Description}

\subsection{Extended Petri Nets}

Our aim is to construct a task-oriented model of the system, with structure and behavior described in the previous section. To achieve it we formulate an extension of Petri nets described as follows. Place/transition Petri net is a quadruple:

$$
\mathbf{P T N}=(\mathbf{P}, \mathbf{T}, \mathbf{A}, \boldsymbol{\Theta}),
$$

where $\mathbf{P}$ is a set of places, $\mathbf{T}$ is a set of transitions, $\mathbf{A} \subseteq \mathbf{P} \times \mathbf{T} \cup \mathbf{T} \times \mathbf{P}$ is a set of arcs, and $\boldsymbol{\Theta} . \mathbf{P} \rightarrow \mathfrak{C}$ is a state function, which assigns to each place $\mathbf{p}$ some nonnegative number $\boldsymbol{\Theta}(\mathbf{p})$ of tokens. A Petri net with an initial state $\boldsymbol{\Theta}$ realizes a process by firing transitions. A transition $\mathbf{t}$ is fireable in a state $\boldsymbol{\Theta}$ (it is written as $\boldsymbol{\Theta} \mathbf{t}\rangle$ ), if each place of its input $\mathbf{I}(\mathbf{t})=\{\mathbf{p} \in \mathbf{P} \mid\langle\mathbf{p t}\rangle \in \mathbf{A}\}$ contains a token:

$$
\Theta[\mathbf{t}\rangle \Leftrightarrow \forall(\mathbf{p} \in \mathbf{I}(\mathbf{t})) \Theta(\mathbf{p})>0 \text {. }
$$

The fired transition $\mathbf{t}$ consumes one token per input place and produces one token in each place of its output $\mathbf{O}(\mathbf{t})=\{\mathbf{p} \in \mathbf{P} \mid\langle\mathbf{t} \mathbf{p}\rangle \in \mathbf{A}\}$, moving the net into the new state $\boldsymbol{\Theta}$ :

$$
\boldsymbol{\Theta}^{\prime}(\mathbf{p})= \begin{cases}\boldsymbol{\Theta}(\mathbf{p})-1 & \text { for } \mathbf{p} \in \mathbf{I}(\mathbf{p}) \backslash \mathbf{O}(\mathbf{p}) \\ \boldsymbol{\Theta}(\mathbf{p})+1 & \text { for } \mathbf{p} \in \mathbf{O}(\mathbf{p}) \mathbf{I}(\mathbf{p}) \\ \boldsymbol{\Theta}(\mathbf{p}) & \text { otherwise }\end{cases}
$$


We introduce following extensions to the above definition:

- Tokens represent data structures defined by the state function of the net model, describing current state of the modeled system. A place $\mathbf{p}$ contains a set $\boldsymbol{\Theta}(\mathbf{p})$ of some nonnegative number of data structures (in particular $\Theta(\mathbf{p})$ can be empty).

- Predicate of a transition $\mathbf{t}$ is a logical function which constrains its fireability, defined on values of data structures of its input $\{\Theta(\mathbf{p}) \mid \mathbf{p} \in \mathbf{I}(\mathbf{t})\}$.

- Action of a fired transition $\mathbf{t}$ transforms its input data to its output data, consuming one token per each input place $\{\boldsymbol{\theta}(\mathbf{p}) \in \boldsymbol{\Theta}(\mathbf{p}) \mid \mathbf{p} \in \mathbf{I}(\mathbf{t})\}$ and producing one token in each output place $\left\{\boldsymbol{\theta}(\mathbf{p})^{\prime} \in \boldsymbol{\Theta}^{\prime}(\mathbf{p}) \mid \mathbf{p} \in \mathbf{O}(\mathbf{t})\right\}$. Information describing actual state of realized process, transformed by actions and transported by tokens, is then used by predicates for deciding about further way of process realisation.

- Timing is a mechanism placing modelled processes in time by means of timestamps inserted to data structures of tokens, time conditions assigned to predicates and time updates performed by actions. A state $\boldsymbol{\Theta}(\tau)$ of the net is defined by sets of tokens with data structures describing contents of places in a moment $\tau$ :

$$
\Theta(\tau)=\{\Theta(\mathbf{p}, \tau) \mid \mathbf{p} \in \mathbf{P}\}
$$

The number of data structures $|\Theta(p, \tau)| \geq 0$ for any $\mathbf{p} \in \mathbf{P}$. More detailed description of timing mechanism and other extensions, applied in somewhat different context of event-oriented models, can be found in [4].

\subsection{Classes of Net Elements}

Petri nets, extended by above definitions, will constitute dynamic discrete event system models, realizing processes of concurrent execution of different kinds of tasks. In order to represent their semantics, we distinguish classes of places and transitions corresponding to the structure and dynamics of the task-oriented system view described in section 2 .

Places will contain tokens with class-specific data structures. Transitions with classspecific inputs and outputs will perform class-specific actions, constrained by classspecific predicates. Semantics of net elements, comprising class-specific terms, is explained together with net model description in section 4.

Task-oriented net model is built of two hierarchical levels. The model consists of two classes of places with tokens representing tasks and resources, and of three classes of "high-level" transitions, i.e. sub-nets (groups of low-level transitions) responsible for generating tasks, executing tasks and providing resources.

High-level class of task executors consists of several classes of low-level transitions, performing alternative behaviors, appropriate to particular cases of the algorithm for system dynamics described in subsection 2.3. The alternatives are realized by means of class-specific, mutually exclusive predicates.

Low-level net structures of remaining two classes of high-level transitions, representing stochastic generators for streams of tasks and states of resources, are not discussed here. Some propositions can be found in [3]. 


\section{Task-Oriented Net Model}

\subsection{General Structure of the Model}

The structure of task-oriented net model, presented on Fig. 2, is quite regular and has three layers of dynamic elements (high-level transitions) separated by two layers of passive elements (places). Model elements of each layer belong to one of five different classes, characterized below in order as they appear on the figure.

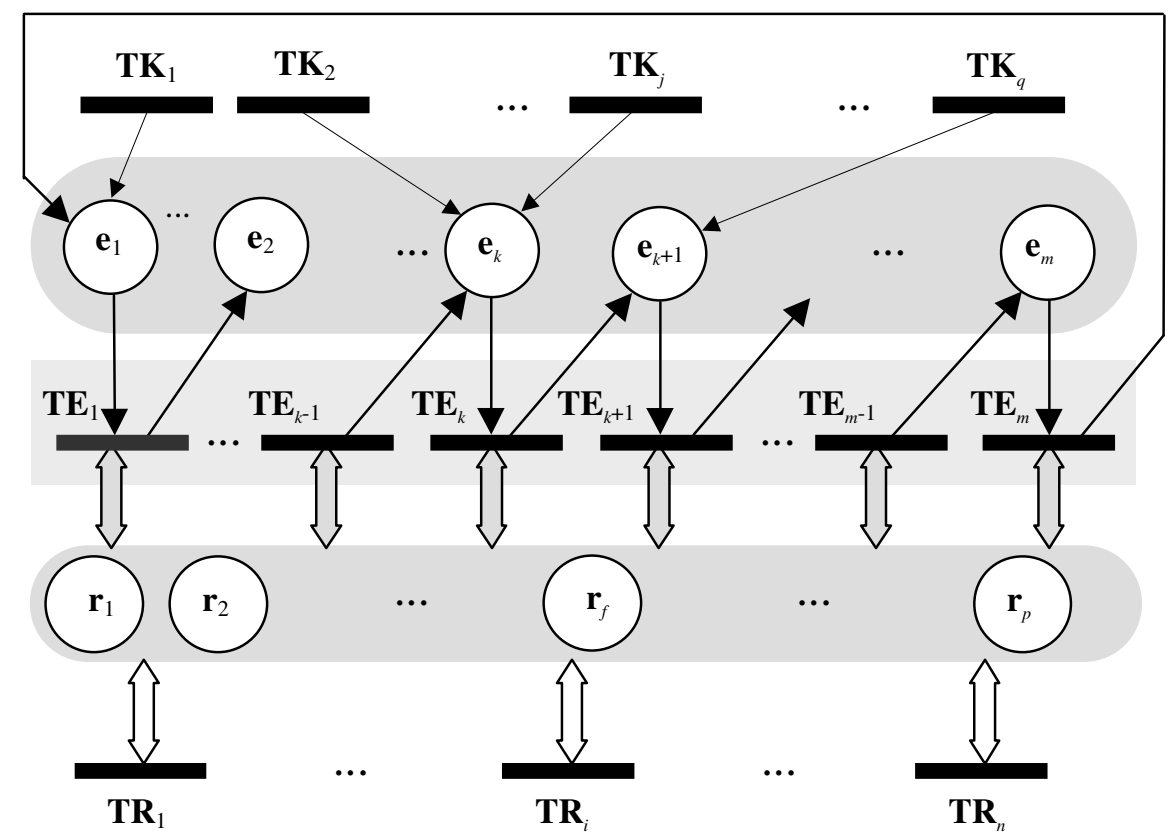

Fig. 2. General structure of the task-oriented net model

Task Generators: a class of transitions corresponding to the set $\mathbf{K}$ is responsible for generating streams of tasks of different kinds. Their actions produce tokens with data structures including descriptions of respective task kinds according to (4) and (5), completed by timestamp of their arrival to the system and by current order value set to 1. Transition $\mathbf{T K}_{j}, j \in\langle 1, \ldots, q\rangle$ is connected to the place $\mathbf{e}_{k}$ with number $k$ such,

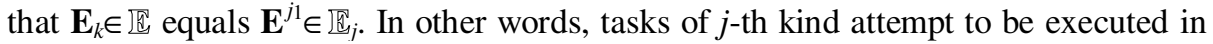
the configuration of the first order (the best for that kind of tasks). The connections showed on Fig. 2 by small black arrows are exemplary.

Tasks: a class of places serving as containers for tasks, waiting for execution in one of the possible configurations of resources. The places $\mathbf{e}_{1}, \ldots, \mathbf{e}_{m}$, corresponding to $m$ executive configurations used by the system, are inputs to transitions responsible for execution of tasks in respective configurations. They hold tokens representing tasks, produced by task generators. 
Task Executors: high-level transitions, corresponding to the family 黑 of all executive configurations used in the system. Transitions of this class consume tasks and perform actions depending on current states of needed resources. They execute tasks in particular configurations if resources are available. If some of needed resources remain in states of inability or inaccessibility, they pass tasks to the input of another task executor, next in the chain of connections showed on the figure by big black arrows. From the other side, transition $\mathbf{T E}_{k}$ is connected with places containing resources of executive configuration $\mathbf{E}_{k}$. Groups of connections $\left\{\mathbf{T E}_{k}\right\} \times \mathbf{E}_{k} \cup \mathbf{E}_{k} \times\left\{\mathbf{T E}_{k}\right\}$ between transitions $\mathbf{T E}_{k}, k=1, \ldots, m$, and their input/output places representing resources of particular executive configurations, are depicted by thick grey arrows on Fig. 2. Sets $\mathbf{E}_{k}, k=1, \ldots, m$ have in general nonempty intersections: a resource place $\mathbf{r}_{f}$ may be connected with several task executors. The detailed structure and function of this class of transitions is described in the subsection 4.2.

Resources: a class of places corresponding to the set $\mathbf{R}$ of system resources (1). These places contain tokens with data including actual states of particular resources (from $1^{\circ}$ to $4^{\circ}$ as enumerated in subsection 2.1), their planned durations and timestamps of last state changes. Tokens representing resources are consumed and "occupied" by task executors.

Resource Providers: a class of transitions corresponding to the family $\mathbb{R}=\mathbf{R}_{1}, \ldots, \mathbf{R}_{n}$ of resource sets (2). They are responsible for current states of resources (except of the state $2^{\circ}$, caused by task executors). Acting concurrently to transitions of other classes, they consume and produce tokens representing resources and temporarily limit their accessibility, possibly in a stochastic manner. An example of detailed realisation of this class of high-level transitions, based on several stochastic generators, can be found in [3]. Groups of input/output connections $\left\{\mathbf{T R}_{i}\right\} \times \mathbf{R}_{i} \cup \mathbf{R}_{i} \times\left\{\mathbf{T R}_{i}\right\}$ between transitions $\mathbf{T R}_{i}, i=1, \ldots, n$ and places representing corresponding subsets of resources, are showed on the figure by thick white arrows. Sets $\mathbf{R}_{i}, i=1, \ldots, n$ are disjoint: each resource place $\mathbf{r}_{f}$ is connected with one particular transition $\mathbf{T R}_{i}$.

Active configuration for a task of $j$-th kind, represented by a token produced by transition $\mathbf{T K}$ and placed on input to transition $\mathbf{T} \mathbf{E}_{k}$, is chosen by a rule similar to the "token ring" principle of communication, applied in computer nets. If $k$-th executive configuration can not be activated for a task waiting for execution in the place $\mathbf{e}_{k}$ (e.g. it is improper for this task, or some of resources in the set $\mathbf{E}_{k}$ are inaccessible), transition $\mathbf{T E}_{k}$ produces a token for that task in another place $\mathbf{e}_{l}$, successive in the closed chain shown on Fig. $2(l=k+1$ for $k<m$ and $l=1$ for $k=m)$. This mechanism is described in the following subsection.

\subsection{Detailed Structure of Task Executors}

A high-level transition of the task executor class forms a subnet, consisting of a group of four parallel low-level transitions of different classes (a), (b), (d) and (e), as shown on Fig. 3. The predicates constraining fireability for each of these transitions are different and mutually exclusive. 

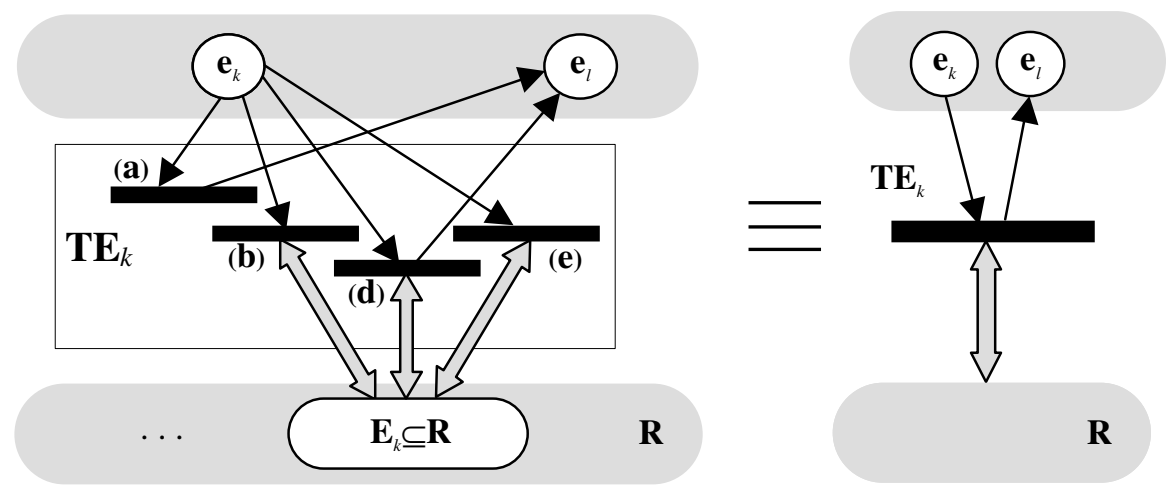

Fig. 3. Detailed structure of the task executor

The place $\mathbf{e}_{k}$ contains tokens representing tasks to be executed in some configurations of resources. Input connection from the place $\mathbf{e}_{k}$ to the high-level transition $\mathbf{T E}_{k}$ is branched to the four parallel low-level transitions with predicates corresponding to the cases distinguished in the algorithm of subsection 2.3. As a consequence, they alternatively consume tasks and perform actions proper to the cases (a), (b), (d) and (e) of the algorithm:

Transition (a) occurs, if an input token in place $\mathbf{e}_{k}$ represents a task of $j$-th kind, which attempts to be executed in some configuration $\mathbf{E}^{j d}$ of order $d$ on the list 宦, different from the configuration $\mathbf{E}_{k}$ bound to the high-level transition $\mathbf{T E}_{k}$. The task is moved to the input of high-level transition $\mathbf{T K}_{l}$, successive in the chain, by consuming the token in place $\mathbf{e}_{k}$ and producing another one with the same data structure in place $\mathbf{e}_{l}$. This action is repeated until the proper executive configuration is reached. Note that the transition (a) has no input resource places.

Transition (b) occurs, when a token in place $\mathbf{e}_{k}$ represents a task attempting to be executed in the configuration $\mathbf{E}_{k}$ and all needed resources are available. It removes a task from the input place and occupies resources by consuming tokens in places of the set $\mathbf{E}_{k}$ and producing new ones in the same places, with states changed to "occupied" and updated values of timestamps and timing functions.

Transition (d) occurs, when a token in place $\mathbf{e}_{k}$ represents a task of $j$-th kind attempting to be executed in the configuration $\mathbf{E}_{k}=\mathbf{E}^{j d}$, which can not be activated because there is at least one token with state $3^{\circ}$ or $4^{\circ}$ in resource places of the set $\mathbf{E}_{k}$. The task is moved, with increased order value, to the input of transition $\mathbf{T K}_{l}$, attempting to be executed in the configuration $\mathbf{E}^{j(d+1)}$, successive on the list $\mathbb{\Perp}_{j}$.

Transition (e) occurs, when a token in place $\mathbf{e}_{k}$ represents a task of $j$-th kind, the configuration $\mathbf{E}_{k}$ is last on the list $\mathbb{\mathbb { Q }}_{j}$ and can not be activated; the task is discarded.

The case (c) of the algorithm is static; waiting for releasing of occupied resources is modeled by timing condition built into the predicate of transition (b). 


\section{Conclusions}

The task-oriented approach for construction and dynamics of net simulation models bases on a set of extended Petri net modeling tools. The modeling method, with its mechanisms for timing, conditional execution and processing of data structures, has been implemented as an object library of net components and proved its usefulness for event-oriented simulation models of transport and logistic systems $[4,5,6]$.

The new, task-oriented view of modeled system was inspired by the reliability behavior of control systems in transport. Such a view permits to define compact models of systems performing tasks by means of distributed resources with limited and dynamically changing accessibility. These system characteristics may be valid for various environments, e.g. for distributed computing and Web based simulation.

Using stochastic generators for streams of tasks and states of resources, this way of simulation modeling, compared with analytical models based on stochastic Petri nets, forms an alternative which is free of limitations on probability distributions.

The presented modeling rules give rise to further evolution in several topics, like multi-directional net structure instead of circular one, stochastic rules of choosing executive configurations in place of simple ordering, special treatment of "refused" tasks etc., which can increase expressive power of this modeling approach. Taking into account the easiness of object implementation of net elements, the described type of models seems promising.

\section{References}

1. Desel J., Reisig W., Place/Transition Petri Nets. In: Lectures on Petri Nets I; Basic Models, Vol. 1491 of LNCS, Springer-Verlag (1998)

2. Ghezzi C., Mandrioli D., Morasca S., Pezzè M.: A General Way to Put Time in Petri Nets. Proceedings of the 5th International Workshop on Software Specification and Design, IEEE-CS Press, Pittsburg (1989)

3. Ochmanska E., Wawrzynski W.: Simulation Model of Control System at Railway Station. Archives of Transport. Polish Academy of Science, Committee of Transport. Warsaw (to appear)

4. Ochmanska E.: System Simulating Technological Processes. ESM'97, Proceedings of the 11th European Simulation Multiconference, Istambul (1997)

5. Ochmanska E.: Object-oriented PN Models with Storage for Transport and Logistic Processes. ESS'97, Proceedings of the 9th European Simulation Symposium ,Simulation in Industry", Passau (1997)

6. Ochmanska E.: Simulation of Serving Trains at Railway Freight Stations. 13th European Simulation Multiconference, Warszawa (1999) 